Jaeyoung Byeon · Zhi-Qiang Wang

\title{
Spherical semiclassical states of a critical frequency for Schrödinger equations with decaying potentials
}

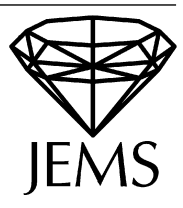

\author{
Dedicated to Professor Antonio Ambrosetti on the occasion of his 60th birthday
}

Received September 15, 2005 and in revised form November 30, 2005

\begin{abstract}
For singularly perturbed Schrödinger equations with decaying potentials at infinity we construct semiclassical states of a critical frequency concentrating on spheres near zeroes of the potentials. The results generalize some recent work of Ambrosetti-Malchiodi-Ni [3] which gives solutions concentrating on spheres where the potential is positive. The solutions we obtain exhibit different behaviors from the ones given in [3].
\end{abstract}

Keywords. Nonlinear Schrödinger equations, critical frequency, concentration on spheres

\section{Introduction}

This paper is concerned with semiclassical states of nonlinear Schrödinger equations with potentials

$$
\left\{\begin{array}{l}
-\varepsilon^{2} \Delta v+V(x) v=v^{p}, \quad x \in \mathbb{R}^{n}, \\
u \in W^{1,2}\left(\mathbb{R}^{n}\right), \quad u>0 .
\end{array}\right.
$$

Here $V \in C\left(\mathbb{R}^{n}, \mathbb{R}\right)$ is a radially symmetric nonnegative potential and $p>1$. In recent years intensive work has been done to construct semiclassical bound states. In particular, following the seminal work by Floer-Weinstein [13], numerous papers have been devoted to constructing various types of spike solutions which concentrate at points of $\mathbb{R}^{n}$. With no intent to survey those results we just refer to the latest monograph [2] for references. Our interest in this paper lies in solutions concentrating on higher dimensional sets, in particular on spheres. In a recent paper [3] Ambrosetti-Malchiodi-Ni constructed solutions concentrating on spheres for equation (1) with positive potentials. The locations of the concentrations are determined by the critical points of a weighted potential. More precisely, if the weighted potential $M(r)=r^{n-1} V^{\ell}(r), \ell=(p+1) /(p-1)-1 / 2$, has a minimum or maximum at some $r^{*}>0$, then (1) has a radial solution $v_{\varepsilon}$ which concentrates on the sphere of radius $r^{*}$. The result was generalized in [5] to the case of decaying potentials. On the other hand, when $\inf _{\mathbb{R}^{n}} V(x)=0$ (this will be referred as

J. Byeon: Department of Mathematics, POSTECH, San 31 Hyoja-dong, Nam-gu, Pohang, Kyungbuk 790-784, Republic of Korea; e-mail: jbyeon@ postech.ac.kr

Z. Q. Wang: Department of Mathematics and Statistics, Utah State University, Logan, UT 84322, USA; e-mail: zhi-qiang.wang@usu.edu 
a critical frequency), spike solutions have been constructed in [9, 10] which concentrate on the zeroes of the potential as $\varepsilon \rightarrow 0$. These solutions are different from the spike solutions and the spherical solutions which concentrate at points and spheres where the potential is positive. In fact the solutions given in [9, 10] are small solutions as $\varepsilon \rightarrow 0$ : the $L^{\infty}$ norm tends to 0 , while the spike and spherical solutions concentrating on points and spheres where the potential is positive have the $L^{\infty}$ norm staying bounded away from zero. We also comment on the recent work in [1, 4, 5] treating potentials which decay to zero at infinity, for which both spike solutions and spherical concentration solutions are constructed, but the concentrations are at positive values of the potentials.

The purpose of the present paper is twofold. First we show that for the critical frequency case we can also construct 'small' solutions concentrating on spheres near zeroes of the potentials. Second, our work covers a general class of decaying potentials for which we construct solutions concentrating near zeroes of the potentials. All of this will be done by further developing and modifying the local minimization techniques of [9]. Set

$$
\mathcal{Z}=\left\{x \in \mathbb{R}^{n} \mid V(x)=0\right\} .
$$

We assume from now on that $V$ satisfies

(V) $\quad V \in C\left(\mathbb{R}^{n}, \mathbb{R}\right)$ is radially symmetric, and $\liminf _{|x| \rightarrow \infty}|x|^{2} V(x) \equiv 4 \lambda>0$.

Note that $(V)$ implies that $\mathcal{Z}$ is bounded. Our main existence result is the following.

Theorem 1. Suppose that $(V)$ holds. Let $A \subset \mathcal{Z}$ be an isolated component of $\mathcal{Z}$ such that $0 \notin A$. Then for $\varepsilon$ sufficiently small, (1) has a radially symmetric solution $v_{\varepsilon} \in W^{1,2}\left(\mathbb{R}^{n}\right)$ such that

$$
\lim _{\epsilon \rightarrow 0}\left\|v_{\varepsilon}\right\|_{\infty}=0 \quad \text { and } \quad \liminf _{\varepsilon \rightarrow 0} \varepsilon^{-2 /(p-1)}\left\|v_{\varepsilon}\right\|_{\infty}>0 .
$$

Moreover, for each $\delta>0$, there are constants $C, c>0$ such that

$$
v_{\varepsilon}(x) \leq C \exp (-c / \varepsilon)\left(\operatorname{dist}\left(x, A^{\delta}\right) / \varepsilon\right)^{\omega_{\varepsilon}},
$$

where

$$
\omega_{\varepsilon} \equiv-\frac{(n-2)+\sqrt{(n-2)^{2}+4 \lambda / \varepsilon^{2}}}{2} \quad \text { and } \quad A^{\delta} \equiv\left\{x \in \mathbb{R}^{n} \mid \operatorname{dist}(x, A) \leq \delta\right\} .
$$

Remark 2. The behavior of the solution $v_{\varepsilon}$ found above depends on the fact that the concentration point is a zero of $V$, and is different from that of solutions constructed in [3]. In [3] if the weighted potential $M(r)=r^{n-1} V(r)^{\ell}, \ell=(p+1) /(p-1)-$ $1 / 2$, has a minimum or maximum at some $r^{*}>0$ then for $\varepsilon$ small a radial solution $v_{\varepsilon}$ concentrates on the sphere of radius $r^{*}$ and $v_{\varepsilon} \sim U\left(\left(r-r^{*}\right) / \varepsilon\right)$, where $U$ is the positive, radial solution of $-U^{\prime \prime}+U=U^{p}$ such that $U^{\prime}(0)=0$. On the other hand, the solutions we give here have different behavior by property (2). More precise information on the asymptotics may depend upon the local behavior of $V$ near $A$ like for the spike solutions in [9]. More precise results will be given in a separate work. 
Remark 3. We point out that, with the decay of the potentials at infinity, the variational problem associated with the equation is not well posed in $W^{1,2}\left(\mathbb{R}^{n}\right)$. In fact, it is not even well posed in the weighted spaces associated naturally to the problem (see [1]). More precisely, the space $E_{\varepsilon}$ defined in Section 2 is not embedded into $L^{p+1}$. Nevertheless, we still manage to construct solutions by a variational method. To overcome the difficulty of dealing with decaying potentials, we devise a new localized approach generalizing the methods of [9].

Remark 4. In [3, 4, 5], a Lyapunov-Schmidt reduction method was used, which requires certain smoothness properties of $V$. In fact, they assume that $V$ and $|\nabla V|$ are bounded. Our approach in this paper is purely variational requiring only the continuity of $V$.

The proof of Theorem 1 is given in Section 2. We finish with a few remarks about further extensions of the results and methods.

\section{Proof of Theorem 1}

The proof of Theorem 1 is based on a minimization process with two constraints which was used in [9] to construct spike solutions concentrating near zeroes of the potential. Here we construct solutions concentrating on spheres near zeroes of $V$.

By a scaling $u(x)=v(\varepsilon x)$ we consider the following equivalent problem:

$$
\left\{\begin{array}{l}
-\Delta u+V(\varepsilon x) u=u^{p}, \quad x \in \mathbb{R}^{n}, \\
u \in W^{1,2}\left(\mathbb{R}^{n}\right), \quad u>0 .
\end{array}\right.
$$

Now, let $A \subset \mathcal{Z}$ be the isolated component as assumed in the theorem. We choose $\delta>0$ such that $0 \notin A^{8 \delta}$, and $A^{8 \delta} \cap\left(\mathcal{Z} \backslash A^{8 \delta}\right)=\emptyset$, where $A^{\delta}=\left\{x \in \mathbb{R}^{n} \mid d(x, A) \leq \delta\right\}$. We set $A_{\varepsilon}^{\delta}=\left\{x \in \mathbb{R}^{n} \mid \varepsilon x \in A^{\delta}\right\}$. Let $C_{0 \text {,rad }}^{\infty}\left(\mathbb{R}^{n}\right)$ be the class of radially symmetric functions in $C_{0}^{\infty}\left(\mathbb{R}^{n}\right)$. Let $E_{\varepsilon}$ the completion of $C_{0, \text { rad }}^{\infty}\left(\mathbb{R}^{n}\right)$ with respect to the norm

$$
\|u\|_{\varepsilon}=\left(\int\left(|\nabla u|^{2}+V(\varepsilon x) u^{2}\right)\right)^{1 / 2} .
$$

We might sometimes use $V_{\varepsilon}$ for $V(\varepsilon x)$.

We first consider the subcritical case, i.e., we assume $1<p<(n+2) /(n-2)$. We will indicate later how to modify the proof to handle the case of $p \geq(n+2) /(n-2)$.

Fix a constant $\gamma$ with $\gamma(p-1) /(p+1)>2$. We define a function $\chi_{\varepsilon}$ by

$$
\chi_{\varepsilon}(x)= \begin{cases}\varepsilon^{-(n-1)-3(p+1) /(p-1)} & \text { if }|x| \leq R_{0} / \varepsilon, x \notin A_{\varepsilon}^{4 \delta} \\ (|x| / \varepsilon)^{\gamma} & \text { if }|x| \geq R_{0} / \varepsilon \\ 0 & \text { if } x \in A_{\varepsilon}^{4 \delta}\end{cases}
$$

Here $R_{0} \geq 1$ is fixed such that $V(x)>0$ for $|x| \geq R_{0}$ and $\mathcal{Z}^{8 \delta} \subset B\left(0, R_{0}\right)$. 
We consider the following minimization problem:

$$
M_{\varepsilon}=\inf \left\{\left.\|u\|_{\varepsilon}^{2}\left|\int_{\mathbb{R}^{n}}\right| u\right|^{p+1} d x=1, \int_{\mathbb{R}^{n}} \chi_{\varepsilon}|u|^{p+1} d x \leq 1, u \in E_{\varepsilon}\right\} .
$$

We note that $\int_{\mathbb{R}^{n}} \chi_{\varepsilon}|u|^{p+1} d x$ may not be well defined on $E_{\varepsilon}$, and may not be differentiable even if $\int_{\mathbb{R}^{n}} \chi_{\varepsilon}|u|^{p+1} d x<\infty$ is defined. We will overcome this deficiency via a certain approximation procedure.

In the following, $C$ denotes a generic constant which may be different on different lines but independent of the limits concerned.

Lemma 5. $\lim _{\varepsilon \rightarrow 0} \varepsilon^{(n-1)(p-1) /(p+1)} M_{\varepsilon}=0$.

Proof. Let $x_{0} \in A$. For all $a>0$ there exists $b>0$ such that $V(x) \leq a$ for all $\left|x-x_{0}\right|$ $\leq b$. Without loss of generality, we can assume $\left|x_{0}\right|=1$ so that $S_{\varepsilon}^{\delta} \subset A_{\varepsilon}^{\delta}$, where $S$ is the unit sphere in $\mathbb{R}^{n}$. Then

$$
\begin{aligned}
& M_{\varepsilon} \leq \inf _{u \in C_{0, \text { rad }}^{\infty}\left(S_{\varepsilon}^{\delta}\right)} \frac{\int\left[|\nabla u|^{2}+V(\varepsilon x) u^{2}\right] d x}{\left(\int_{\mathbb{R}^{n}}|u|^{p+1} d x\right)^{2 /(p+1)}} \leq \inf _{u \in C_{0, \text { rad }}^{\infty}\left(S_{\varepsilon}^{\delta}\right)} \frac{\int\left[|\nabla u|^{2}+a u^{2}\right] d x}{\left(\int_{\mathbb{R}^{n}}|u|^{p+1} d x\right)^{2 /(p+1)}} \\
& \leq C \varepsilon^{-(n-1)(p-1) /(p+1)} \inf _{u \in C_{0}^{\infty}(-\delta / \varepsilon, \delta / \varepsilon)} \frac{\int_{-\delta / \varepsilon}^{\delta / \varepsilon}\left[\left|u^{\prime}\right|^{2}+a u^{2}\right] d r}{\left(\int_{-\delta / \varepsilon}^{\delta / \varepsilon}|u|^{p+1} d r\right)^{2 /(p+1)}} .
\end{aligned}
$$

If we let $\varepsilon \rightarrow 0$, the last infimum is bounded by a constant which tends to zero as $a \rightarrow 0$. Since $a$ is arbitrary, the lemma follows.

Lemma 6. For $\varepsilon$ small, $M_{\varepsilon}$ is achieved at $u_{\varepsilon}$ which satisfies for some $\alpha_{\varepsilon} \geq 0 \geq \beta_{\varepsilon}$,

$$
-\Delta u_{\varepsilon}+V(\varepsilon x) u_{\varepsilon}=\alpha_{\varepsilon}\left(u_{\varepsilon}\right)^{p}+\beta_{\varepsilon} \chi_{\varepsilon}\left(u_{\varepsilon}\right)^{p}, \quad u_{\varepsilon}>0 .
$$

Proof. In order to show that $M_{\varepsilon}$ is achieved we use approximations. For a fixed $\varepsilon>0$, we choose $R_{m}>0$ such that $R_{0} / \varepsilon<R_{1}<R_{2}<\cdots$ and $\lim _{m \rightarrow \infty} R_{m}=\infty$. Define $E_{\varepsilon}^{m} \equiv E_{\varepsilon} \cap W_{0}^{1,2}\left(B\left(0, R_{m}\right)\right)$. Then we consider a restricted minimization problem

$$
M_{\varepsilon}^{m}=\inf \left\{\left.\|u\|_{\varepsilon}^{2}\left|\int_{\mathbb{R}^{n}}\right| u\right|^{p+1} d x=1, \int_{\mathbb{R}^{n}} \chi_{\varepsilon}|u|^{p+1} d x \leq 1, u \in E_{\varepsilon}^{m}\right\} .
$$

It is standard to show that there exists a nonnegative minimizer $u_{\varepsilon}^{m}$ of $M_{\varepsilon}^{m}$, that $M_{\varepsilon}^{m} \geq M_{\varepsilon}$ and $M_{\varepsilon}^{m} \rightarrow M_{\varepsilon}$ as $m \rightarrow \infty$. Thus, $\left\{u_{\varepsilon}^{m}\right\}_{m}$ is a minimizing sequence for $M_{\varepsilon}$, and for some $\alpha_{\varepsilon}^{m}, \beta_{\varepsilon}^{m} \in \mathbb{R}, u_{\varepsilon}^{m}$ satisfies

$$
-\Delta u_{\varepsilon}^{m}+V(\varepsilon x) u_{\varepsilon}^{m}=\alpha_{\varepsilon}^{m}\left(u_{\varepsilon}^{m}\right)^{p}+\beta_{\varepsilon}^{m} \chi_{\varepsilon}\left(u_{\varepsilon}^{m}\right)^{p}, \quad u_{\varepsilon}^{m}>0 \text { in } B\left(0, R_{m}\right) .
$$

Taking a subsequence if necessary, we can assume that for some $u_{\varepsilon} \in E_{\varepsilon}, u_{\varepsilon}^{m}$ converges weakly to $u_{\varepsilon}$ in $E_{\varepsilon}$ as $m \rightarrow \infty$. Since $\int_{\mathbb{R}^{n}} \chi_{\varepsilon}\left|u_{\varepsilon}^{m}\right|^{p+1} d x \leq 1$, it follows that for any fixed large $R>0$,

$$
\int_{\mathbb{R}^{n} \backslash B(0, R)}\left|u_{\varepsilon}^{m}\right|^{p+1} d x \leq(\varepsilon / R)^{\gamma} .
$$


For $1<p<(n+2) /(n-2)$, the embedding of $E_{\varepsilon}^{m}$ into $L^{p+1}\left(B\left(0, R_{m}\right)\right)$ is compact. Thus it follows that $\int_{\mathbb{R}^{n}}\left(u_{\varepsilon}\right)^{p+1} d x=1$, and that $\int_{B(0, T)} \chi_{\varepsilon}\left|u_{\varepsilon}\right|^{p+1} d x \leq 1$ for each $T>0$. Note that $\left\|u_{\varepsilon}\right\|_{\varepsilon} \leq \liminf _{m \rightarrow \infty}\left\|u_{\varepsilon}^{m}\right\|_{\varepsilon}$. This implies that $u_{\varepsilon}$ is a minimizer of $M_{\varepsilon}>0$.

In equation [8], we can show as in [9] that $\alpha_{\varepsilon}^{m} \geq 0 \geq \beta_{\varepsilon}^{m}$.

Next we show $u_{\varepsilon}$ satisfies equation (6). We claim that for $0<\varepsilon<1 / 2,\left\{\alpha_{\varepsilon}^{m}\right\}_{m}$ is bounded. In fact, arguing by contradiction, assume that $\lim \sup _{m \rightarrow \infty} \alpha_{\varepsilon}^{m}=\infty$ for some $0<\varepsilon<1 / 2$. Without loss of generality, we may assume that $\lim _{m \rightarrow \infty} \alpha_{\varepsilon}^{m}=\infty$. For any $\sigma>0$, we choose $\phi_{\sigma} \in C_{0}^{\infty}\left(\operatorname{int}\left(A_{\varepsilon}^{4 \delta}\right)\right)$ satisfying $0 \leq \phi_{\sigma} \leq 1, \phi_{\sigma}(x)=1$ for $\operatorname{dist}\left(x, \partial A_{\varepsilon}^{4 \delta}\right) \geq \sigma$, and $\left|\nabla \phi_{\sigma}\right| \leq 2 / \sigma$. From (8), we deduce that

$$
\int_{\mathbb{R}^{n}}\left(\left|\nabla u_{\varepsilon}^{m}\right|^{2} \phi_{\sigma}+\nabla u_{\varepsilon}^{m} \cdot \nabla \phi_{\sigma} u_{\varepsilon}^{m}+V_{\varepsilon}\left(u_{\varepsilon}^{m}\right)^{2} \phi_{\sigma}\right) d x=\alpha_{\varepsilon}^{m} \int_{\mathbb{R}^{n}} \phi_{\sigma}\left(u_{\varepsilon}^{m}\right)^{p+1} d x .
$$

Since $\inf _{x \in \operatorname{supp}\left(\left|\nabla \phi_{\sigma}\right|\right)} V_{\varepsilon}(x)>0$, it follows that for some $C>0$, independent of $m$,

$$
\int_{\mathbb{R}^{n}}\left(\left|\nabla u_{\varepsilon}^{m}\right|^{2} \phi_{\sigma}+\nabla u_{\varepsilon}^{m} \cdot \nabla \phi_{\sigma} u_{\varepsilon}^{m}+V_{\varepsilon}\left(u_{\varepsilon}^{m}\right)^{2} \phi_{\sigma}\right) d x \leq C\left\|u_{\varepsilon}^{m}\right\|_{\varepsilon}^{2} .
$$

Since $\left\{\left\|u_{\varepsilon}^{m}\right\|_{\varepsilon}\right\}_{m}$ is bounded and $\lim _{m \rightarrow \infty} \alpha_{\varepsilon}^{m}=\infty$, we see that for each $\sigma>0$, $\lim _{m \rightarrow \infty} \int_{\mathbb{R}^{n}} \phi_{\sigma}\left(u_{\varepsilon}^{m}\right)^{p+1} d x=0$. By the constraints on $u_{\varepsilon}^{m}$ we have for $\sigma>0$,

$$
\liminf _{m \rightarrow \infty} \int_{\left\{x \in \mathbb{R}^{n} \mid \operatorname{dist}\left(x, \partial A_{\varepsilon}^{4 \delta}\right) \leq \sigma\right\}}\left(u_{\varepsilon}^{m}\right)^{p+1} d x>0 .
$$

Since $\lim _{m \rightarrow \infty} \int_{\mathbb{R}^{n}} \phi_{\sigma}\left(u_{\varepsilon}^{m}\right)^{p+1} d x=0$ for each $\sigma>0$, there exist $x_{m} \in A_{\varepsilon}^{4 \delta}$ such that $\lim _{m \rightarrow \infty} \operatorname{dist}\left(x_{m}, \partial A_{\varepsilon}^{4 \delta}\right)=0$ and $u_{m}\left(x_{m}\right)=1$. Taking a subsequence if necessary, we may assume that $\lim _{m \rightarrow \infty}\left|x_{m}\right|=r_{0}$ and for each $\sigma>0$,

$$
\liminf _{m \rightarrow \infty} \int_{\left\{x\left|r_{0}-\sigma \leq\right| x \mid \leq r_{0}+\sigma\right\}}\left(u_{\varepsilon}^{m}\right)^{p+1} d x>0 .
$$

We define $D_{r_{0}}^{\sigma} \equiv\left\{x\left|r_{0}-\sigma \leq\right| x \mid \leq r_{0}+\sigma\right\}$. By the Poincaré inequality, there exists some $C>0$, independent of $\sigma$, such that for sufficiently large $m>0$,

$$
\int_{D_{r_{0}}^{\sigma}}\left(u_{\varepsilon}^{m}-1\right)_{+}^{2} d x \leq C \sigma^{2} \int_{D_{r_{0}}^{\sigma}}\left(\left|\nabla\left(u_{\varepsilon}^{m}-1\right)_{+}\right|^{2}+V_{\varepsilon}\left(u_{\varepsilon}^{m}-1\right)_{+}^{2}\right) d x .
$$

Note that

$$
\int_{D_{r_{0}}^{\sigma}}\left(\left|\nabla\left(u_{\varepsilon}^{m}-1\right)_{+}\right|^{2}+V_{\varepsilon}\left(u_{\varepsilon}^{m}-1\right)_{+}^{2}\right) d x \leq \int_{D_{r_{0}}^{\sigma}}\left(\left|\nabla u_{\varepsilon}^{m}\right|^{2}+V_{\varepsilon}\left(u_{\varepsilon}^{m}\right)^{2}\right) d x .
$$


Then, by (10), 11], the Hölder inequality and Sobolev inequality, we see that for some $s \in(0,1)$ and $C>0$,

$$
\begin{aligned}
\int_{D_{r_{0}}^{\sigma}}\left(u_{\varepsilon}^{m}-1\right)_{+}^{p+1} d x \leq & C\left(\int_{D_{r_{0}}^{\sigma}}\left(u_{\varepsilon}^{m}-1\right)_{+}^{2} d x\right)^{s(p+1) / 2} \\
& \times\left(\int_{D_{r_{0}}^{\sigma}}\left(\left|\nabla\left(u_{\varepsilon}^{m}-1\right)_{+}\right|^{2}+V_{\varepsilon}\left(u_{\varepsilon}^{m}-1\right)_{+}^{2}\right) d x\right)^{(1-s)(p+1) / 2} \\
\leq & C \sigma^{s(p+1)}\left\|u_{\varepsilon}^{m}\right\|_{\varepsilon}^{p+1} .
\end{aligned}
$$

This contradicts $(9\}$ since $\left\{\left\|u_{\varepsilon}^{m}\right\|_{\varepsilon}\right\}_{m}$ is bounded. Thus we see that $\left\{\alpha_{\varepsilon}^{m}\right\}_{m}$ is bounded.

Finally, for any radially symmetric function $\varphi \in C_{0}^{\infty}\left(B(0, R / \varepsilon) \backslash A_{\varepsilon}^{4 \delta}\right)$, we have

$$
\int_{\mathbb{R}^{n}}\left(\nabla u_{\varepsilon}^{m} \cdot \nabla \varphi+V_{\varepsilon} u_{\varepsilon}^{m} \varphi\right) d x=\alpha_{\varepsilon}^{m} \int_{\mathbb{R}^{n}}\left(u_{\varepsilon}^{m}\right)^{p} \varphi d x
$$

Since $u_{\varepsilon}^{m}$ converges weakly to $u_{\varepsilon}$ in $E_{\varepsilon}$ as $m \rightarrow \infty$, it follows that $\alpha_{\varepsilon}^{m}$ converges to some $\alpha_{\varepsilon} \geq 0$ as $m \rightarrow \infty$. Then, since for any $\varphi \in C_{0, \text { rad }}^{\infty}\left(\mathbb{R}^{n}\right)$,

$$
\int_{\mathbb{R}^{n}}\left(\nabla u_{\varepsilon}^{m} \cdot \nabla \varphi+V_{\varepsilon} u_{\varepsilon}^{m} \varphi\right) d x=\alpha_{\varepsilon}^{m} \int_{\mathbb{R}^{n}}\left(u_{\varepsilon}^{m}\right)^{p} \varphi d x+\beta_{\varepsilon}^{m} \int_{\mathbb{R}^{n}} \chi_{\varepsilon}\left(u_{\varepsilon}^{m}\right)^{p} \varphi d x,
$$

we see that $\lim _{m \rightarrow \infty} \beta_{\varepsilon}^{m}=\beta_{\varepsilon}$ for some $\beta_{\varepsilon} \leq 0$. Now, it follows that for some $\alpha_{\varepsilon} \geq 0$ and $\beta_{\varepsilon} \leq 0$,

$$
-\Delta u_{\varepsilon}+V(\varepsilon x) u_{\varepsilon}=\alpha_{\varepsilon} u_{\varepsilon}^{p}+\beta_{\varepsilon} \chi_{\varepsilon} u_{\varepsilon}^{p}, \quad u_{\varepsilon}>0
$$

We will show that for $\varepsilon$ small

$$
\int_{\mathbb{R}^{n}} \chi_{\varepsilon}\left|u_{\varepsilon}\right|^{p+1} d x<1
$$

If this is the case, for any $\varphi \in C_{0, \mathrm{rad}}^{\infty}\left(\mathbb{R}^{n}\right)$, we define

$$
\varphi_{s} \equiv\left(u_{\varepsilon}+s \varphi\right)\left(\int_{\mathbb{R}^{n}}\left(u_{\varepsilon}+s \varphi\right)^{p+1} d x\right)^{-1 /(p+1)}
$$

Then we see that $\varphi_{0}=u_{\varepsilon}, \int_{\mathbb{R}^{n}}\left(\varphi_{s}\right)^{p+1} d x=1$ and that $\int_{\mathbb{R}^{n}} \chi_{\varepsilon}\left(\varphi_{s}\right)^{p+1} d x<1$ for small $|s|$. Thus we deduce that

$$
0=\left.\frac{d\left\|\varphi_{s}\right\|_{\varepsilon}^{2}}{d s}\right|_{s=0}=\int_{\mathbb{R}^{n}}\left(\nabla u_{\varepsilon} \cdot \nabla \varphi+V_{\varepsilon} u_{\varepsilon} \varphi\right) d x-\left\|u_{\varepsilon}\right\|_{\varepsilon}^{2} \int_{\mathbb{R}^{n}}\left(u_{\varepsilon}\right)^{p} \varphi d x .
$$

This implies that

$$
-\Delta u_{\varepsilon}+V_{\varepsilon} u_{\varepsilon}=M_{\varepsilon}\left(u_{\varepsilon}\right)^{p}, \quad u_{\varepsilon}>0 \quad \text { in } \mathbb{R}^{n}
$$


Then $w_{\varepsilon}=\left(M_{\varepsilon}\right)^{1 / p-1} u_{\varepsilon}$ is a solution of

$$
-\Delta w_{\varepsilon}+V(\varepsilon x) w_{\varepsilon}=\left(w_{\varepsilon}\right)^{p}, \quad w_{\varepsilon}>0 \quad \text { in } \mathbb{R}^{n},
$$

and $v_{\varepsilon}(x):=w_{\varepsilon}\left(\varepsilon^{-1} x\right)=\left(M_{\varepsilon}\right)^{1 / p-1} u_{\varepsilon}\left(\varepsilon^{-1} x\right)$ solves (1).

To show $\int_{\mathbb{R}^{n}} \chi_{\varepsilon}\left|u_{\varepsilon}\right|^{p+1} d x<1$, we need some asymptotic properties of $\alpha_{\varepsilon}$ and $u_{\varepsilon}$ given in Lemmas 7 and 8

Lemma 7. In the previous notations, one has $\lim _{\varepsilon \rightarrow 0} \varepsilon^{(n-1)(p-1) /(p+1)} \alpha_{\varepsilon}=0$.

Proof. To the contrary, assume, taking a subsequence if necessary, that $\lim _{\varepsilon \rightarrow 0} \varepsilon^{(n-1)(p-1) /(p+1)} \alpha_{\varepsilon} \equiv \alpha \in(0, \infty]$. In the previous notation one has $\phi_{\sigma} \in$ $C_{0}^{\infty}\left(\operatorname{int}\left(A_{\varepsilon}^{4 \delta}\right)\right)$ satisfying $0 \leq \phi_{\sigma} \leq 1, \phi_{\sigma}(x)=1$ for $\operatorname{dist}\left(x, \partial A_{\varepsilon}^{4 \delta}\right) \geq \sigma$, and $\left|\nabla \phi_{\sigma}\right| \leq$ $2 / \sigma$. From equation (6), we deduce that

$$
\int_{\mathbb{R}^{n}}\left(\left|\nabla u_{\varepsilon}\right|^{2} \phi_{\sigma}+\nabla u_{\varepsilon} \cdot \nabla \phi_{\sigma} u_{\varepsilon}+V_{\varepsilon}\left(u_{\varepsilon}\right)^{2} \phi_{\sigma}\right) d x=\alpha_{\varepsilon} \int_{\mathbb{R}^{n}} \phi_{\sigma}\left(u_{\varepsilon}\right)^{p+1} d x .
$$

Since $\inf _{\text {supp }\left(\left|\nabla \phi_{\sigma}\right|\right)} V_{\varepsilon}(x)>0$, it follows that for some $C>0$, independent of $\varepsilon$,

$$
\int_{\mathbb{R}^{n}}\left(\left|\nabla u_{\varepsilon}\right|^{2} \phi_{\sigma}+\nabla u_{\varepsilon} \cdot \nabla \phi_{\sigma} u_{\varepsilon}+V_{\varepsilon}\left(u_{\varepsilon}\right)^{2} \phi_{\sigma}\right) d x \leq C\left\|u_{\varepsilon}\right\|_{\varepsilon}^{2} .
$$

Since $\lim _{\varepsilon \rightarrow 0} \varepsilon^{(n-1)(p-1) /(p+1)}\left\|u_{\varepsilon}\right\|_{\varepsilon}^{2}=0$ and $\lim _{\varepsilon \rightarrow \infty} \varepsilon^{(n-1)(p-1) /(p+1)} \alpha_{\varepsilon}>0$, for each $\sigma>0, \lim _{\varepsilon \rightarrow \infty} \int_{\mathbb{R}^{n}} \phi_{\sigma}\left(u_{\varepsilon}\right)^{p+1} d x=0$. Since $\int_{\mathbb{R}^{n}} \chi_{\varepsilon}\left(u_{\varepsilon}\right)^{p+1} d x \leq 1$, it follows that for any $\sigma>0$ we have

$$
\lim _{\varepsilon \rightarrow 0} \int_{\left\{x \in \mathbb{R}^{n} \mid \operatorname{dist}\left(x, \partial A_{\varepsilon}^{4 \delta}\right) \geq \sigma\right\}}\left(u_{\varepsilon}\right)^{p+1} d x=0 .
$$

Thus, there exist some $x_{0} \in \partial A_{\varepsilon}^{4 \delta}$ and $\omega>0$ such that for any $\sigma>0$,

$$
\liminf _{\varepsilon \rightarrow 0} \int_{\left\{x \in \mathbb{R}^{n}|| x_{0}|/ \varepsilon-\sigma \leq| x|\leq| x_{0} \mid / \varepsilon+\sigma\right\}}\left(u_{\varepsilon}\right)^{p+1} d x \geq 2 \omega .
$$

We fix $\sigma>0$ and choose a radially symmetric $\psi_{\sigma} \in C_{0}^{\infty}$ such that

$$
\psi_{\sigma}(x)= \begin{cases}0 & \text { if }|| x|-| x_{0}|/ \varepsilon| \geq 2 \sigma, \\ 1 & \text { if }|| x|-| x_{0}|/ \varepsilon| \leq \sigma\end{cases}
$$

$0 \leq \psi_{\sigma} \leq 1$ and $\left|\nabla \psi_{\sigma}\right| \leq 3 / \sigma$. Then $\liminf _{\varepsilon \rightarrow 0} \int_{\mathbb{R}^{n}}\left(\psi_{\sigma} u_{\varepsilon}\right)^{p+1} d x \geq \omega$.

On the other hand, we claim that

$$
\lim _{\varepsilon \rightarrow 0} \varepsilon^{(n-1) p-1 / p+1}\left\|\psi_{\sigma} u_{\varepsilon}\right\|_{\varepsilon}^{2}=0 .
$$

This follows from Lemma 5 and the fact that for some $C>0$, independent of $\varepsilon>0$,

$$
\left\|\psi_{\sigma} u_{\varepsilon}\right\|_{\varepsilon}^{2} \leq C\left\|u_{\varepsilon}\right\|_{\varepsilon}^{2}=C M_{\varepsilon}
$$

here we used the fact that $a_{0}:=\inf _{\operatorname{supp}\left(\psi_{\sigma}\right)} V_{\varepsilon}>0$. 
Finally, setting $D(\varepsilon) \equiv\left\{x \in \mathbb{R}^{n}|| x_{0}|/ \varepsilon-2 \sigma \leq| x|\leq| x_{0} \mid / \varepsilon+2 \sigma\right\}$, we deduce

$$
\begin{aligned}
\liminf _{\varepsilon \rightarrow 0} \varepsilon^{(n-1)(p-1) /(p+1)}\left\|\psi_{\sigma} u_{\varepsilon}\right\|_{\varepsilon}^{2} & \\
& \geq \lim _{\varepsilon \rightarrow 0} \varepsilon^{(n-1)(p-1) /(p+1)}\left\|\psi_{\sigma} u_{\varepsilon}\right\|_{p+1}^{2} \inf _{u \in C_{0, \text { rad }}^{1}(D(\varepsilon))} \frac{\|u\|_{\varepsilon}^{2}}{\|u\|_{p+1}^{2}} \\
& \geq \omega^{2 /(p+1)} \liminf _{\varepsilon \rightarrow 0}\left(\left|x_{0}\right|-2 \varepsilon \sigma\right)^{n-1}\left(\left|x_{0}\right|+2 \varepsilon \sigma\right)^{-(n-1) 2 /(p+1)} J_{\sigma},
\end{aligned}
$$

where

$$
J_{\sigma} \equiv \inf _{g \in C_{0}^{1}((-2 \sigma, 2 \sigma))} \frac{\int_{-2 \sigma}^{2 \sigma}\left(\left|g^{\prime}(s)\right|^{2}+a_{0} g(s)^{2}\right) d s}{\left(\int_{-2 \sigma}^{2 \sigma} g(s)^{p+1} d s\right)^{2 /(p+1)}} .
$$

This implies that

$$
\liminf _{\varepsilon \rightarrow 0} \varepsilon^{(n-1)(p-1) /(p+1)}\left\|\psi_{\sigma} u_{\varepsilon}\right\|_{\varepsilon}^{2} \geq \omega^{2 /(p+1)}\left|x_{0}\right|^{(n-1)(p-1) /(p+1)} J_{\sigma}>0,
$$

which contradicts 15 . This completes the proof.

Lemma 8. If $u_{\varepsilon}$ and $\alpha_{\varepsilon}$ are as above, then

$$
\lim _{\varepsilon \rightarrow 0}\left\|\left(\alpha_{\varepsilon}\right)^{1 /(p-1)} u_{\varepsilon}\right\|_{L^{\infty}\left(\mathbb{R}^{n}\right)}=0 .
$$

Proof. We note that

$$
-\Delta\left(\left(\alpha_{\varepsilon}\right)^{1 /(p-1)} u_{\varepsilon}\right)+V(\varepsilon x)\left(\left(\alpha_{\varepsilon_{n}}\right)^{1 /(p-1)} u_{\varepsilon}\right) \leq\left(\left(\alpha_{\varepsilon}\right)^{1 /(p-1)} u_{\varepsilon}\right)^{p} \quad \text { on } \mathbb{R}^{n} .
$$

Suppose that $\liminf _{\varepsilon \rightarrow 0} \int_{B(y / \varepsilon, 2)}\left(\left(\alpha_{\varepsilon}\right)^{1 /(p-1)} u_{\varepsilon}\right)^{p+1} d x>0$ for some $y \in \mathbb{R}^{n} \backslash\{0\}$. This implies that

$$
\liminf _{\varepsilon \rightarrow 0}(\varepsilon /|y|)^{n-1}\left(\alpha_{\varepsilon}\right)^{(p+1) /(p-1)} \int_{\left\{x \in \mathbb{R}^{n}|| y|/ \varepsilon-2 \leq| x|\leq| y \mid / \varepsilon+2\right\}}\left(u_{\varepsilon}\right)^{p+1} d x>0 .
$$

This formula contradicts Lemma 7 Thus, we see that

$$
\limsup _{\varepsilon \rightarrow 0} \int_{B(y / \varepsilon, 2)}\left(\left(\alpha_{\varepsilon}\right)^{1 /(p-1)} u_{\varepsilon}\right)^{p+1} d x=0 .
$$

Then, by the Sobolev embedding and a Moser iteration argument, we deduce that

$$
\lim _{\varepsilon \rightarrow 0}\left\|\left(\alpha_{\varepsilon}\right)^{1 /(p-1)} u_{\varepsilon}\right\|_{L^{\infty}\left(\left\{x \in \mathbb{R}^{n}|| y|/ \varepsilon-1 \leq| x|\leq| y \mid / \varepsilon+1\right\}\right)}=0 .
$$

Since $0 \notin A^{4 \delta}$ and $\int_{\mathbb{R}^{n}} \chi_{\varepsilon}\left(u_{\varepsilon}\right)^{p+1} d x \leq 1$, there exists a constant $r_{0}>0$ such that for small $\varepsilon>0$,

$$
\int_{B\left(0,2 r_{0} / \varepsilon\right)}\left(u_{\varepsilon}\right)^{p+1} d x \leq \varepsilon^{(n-1)+3(p+1) /(p-1)} .
$$


This implies that for small $\varepsilon>0$,

$$
\int_{B\left(0,2 r_{0} / \varepsilon\right)}\left(\left(\alpha_{\varepsilon}\right)^{1 /(p-1)} u_{\varepsilon}\right)^{p+1} d x \leq \varepsilon^{3(p+1) /(p-1)} .
$$

Then, again by standard results, we deduce that

$$
\lim _{\varepsilon \rightarrow 0}\left\|\left(\alpha_{\varepsilon}\right)^{1 /(p-1)} u_{\varepsilon}\right\|_{L^{\infty}\left(B\left(0, r_{0} / \varepsilon\right)\right)}=0 .
$$

Proof of the main theorem. Define $w_{\varepsilon} \equiv\left(\alpha_{\varepsilon}\right)^{1 /(p-1)} u_{\varepsilon}$. Then

$$
-\Delta w_{\varepsilon}+V_{\varepsilon} w_{\varepsilon} \leq\left(w_{\varepsilon}\right)^{p} \quad \text { on } \mathbb{R}^{n} \text {. }
$$

By Lemma 8 , we see that $\left\|w_{\varepsilon}\right\|_{L^{\infty}} \rightarrow 0$ as $\varepsilon \rightarrow 0$. We let

$$
2 c \equiv \inf _{x \in B\left(0,3 R_{0}\right) \backslash \mathcal{Z}^{\delta}} V(x) .
$$

Then, by a comparison principle, we deduce (see [9]) that for small $\varepsilon>0$,

$$
w_{\varepsilon} \leq \exp \left(-c \operatorname{dist}\left(x, \partial\left(B\left(0,3 R_{0} / \varepsilon\right) \backslash \mathcal{Z}_{\varepsilon}^{\delta}\right)\right)\right)
$$

Thus, we see that $\max _{x \in \partial \mathcal{Z}_{\varepsilon}^{2 \delta}} w_{\varepsilon}(x) \leq \exp (-c \delta / \varepsilon)$ for small $\varepsilon>0$.

For a connected component $K$ of $\operatorname{int}\left(\mathcal{Z}^{4 \delta} \backslash A^{4 \delta}\right)$, we consider the first eigenvalue problem on $K$,

$$
\left\{\begin{array}{l}
\Delta \Phi+\lambda_{1} \Phi=0, \quad x \in K \\
\Phi(x)=0, \quad x \in \partial K
\end{array}\right.
$$

Define $\Phi_{\varepsilon}(x) \equiv \Phi(\varepsilon x)$. We may assume that $\max _{x \in K \cap \partial \mathcal{Z}^{3 \delta}} \Phi(x) \geq 1$. By elliptic estimates [14, Theorem 9.20] and from the fact that $\int_{\mathbb{R}^{n}} \chi_{\varepsilon}\left(w_{\varepsilon}\right)^{p+1} d x \leq\left(\alpha_{\varepsilon}\right)^{(p+1) /(p-1)}$, it follows that $\left\|w_{\varepsilon}\right\|_{L^{\infty}\left(\mathcal{Z}_{\varepsilon}^{3 \delta} \backslash A_{\varepsilon}^{3 \delta}\right)} \leq C \varepsilon^{3 /(p-1)}$ for some $C>0$. Then, for sufficiently small $\varepsilon>0$,

$$
-\Delta \Phi_{\varepsilon}+V_{\varepsilon} \Phi_{\varepsilon} \geq\left(w_{\varepsilon}\right)^{p-1} \Phi_{\varepsilon} \quad \text { in } K_{\varepsilon} .
$$

By the comparison principle, we see that

$$
w_{\varepsilon}(x) \leq \exp (-c \delta / \varepsilon) \Phi_{\varepsilon}(x) \quad \text { for } x \in K_{\varepsilon} \cap \mathcal{Z}_{\varepsilon}^{3 \delta} .
$$

Thus, we conclude that for some $C, c>0$,

$$
\left\|w_{\varepsilon}(x)\right\|_{L^{\infty}\left(B\left(0,3 R_{0} / \varepsilon\right) \backslash A_{\varepsilon}^{4 \delta}\right)} \leq C \exp (-c \delta / \varepsilon) .
$$

From the inequality $\int_{\mathbb{R}^{n}} \chi_{\varepsilon}\left(w_{\varepsilon}\right)^{p+1} d x \leq\left(\alpha_{\varepsilon}\right)^{(p+1) /(p-1)}$, it follows that there exists $C>0$ such that for any $y \in \mathbb{R}^{n} \backslash B(0,2 R / \varepsilon)$,

$$
\int_{B(y, 2)}\left(w_{\varepsilon}\right)^{p+1} d x \leq C\left(\frac{\varepsilon}{R_{0}}\right)^{n-1}\left(\alpha_{\varepsilon}\right)^{(p+1) /(p-1)}\left(\frac{\varepsilon}{|y|}\right)^{\gamma} \leq C\left(\frac{\varepsilon}{|y|}\right)^{\gamma} .
$$


Thus, [14, Theorem 9.20] shows that for some $C>0$ independent of $y$ we have $w_{\varepsilon}(x) \leq$ $C(\varepsilon /|x|)^{\gamma /(p+1)}$ for any $x \in B(y, 1)$. We define

$$
\omega_{\varepsilon} \equiv-\frac{(n-2)+\sqrt{(n-2)^{2}+4 \lambda / \varepsilon^{2}}}{2} .
$$

Then, setting $\psi_{\varepsilon}(r)=r^{\omega_{\varepsilon}}$, we deduce from condition $(V)$ that for small $\varepsilon>0$,

$$
-\Delta \psi_{\varepsilon}+V_{\varepsilon} \psi_{\varepsilon} \geq\left(\frac{2 \lambda}{\varepsilon^{2}}-\omega_{\varepsilon}^{2}-(n-2) \omega_{\varepsilon}\right) r^{\omega_{\varepsilon}-2} \geq \frac{\lambda}{\varepsilon^{2}} r^{\omega_{\varepsilon}-2}, \quad r \geq R_{0} / \varepsilon
$$

Thus, it follows that for small $\varepsilon>0$,

$$
-\Delta \psi_{\varepsilon}+V_{\varepsilon} \psi_{\varepsilon} \geq\left(w_{\varepsilon}\right)^{p-1} \psi_{\varepsilon} \quad \text { in } \mathbb{R}^{n} \backslash B\left(0,2 R_{0} / \varepsilon\right)
$$

Note that $\max _{x \in \partial B\left(0,2 R_{0} / \varepsilon\right)} w_{\varepsilon}(x) \leq C \exp (-c / \varepsilon)$ for some $C, c>0$. Then, by the maximum principle, we find that for some $C, c>0$,

$$
w_{\varepsilon}(x) \leq C \exp (-c / \varepsilon) \psi_{\varepsilon}(x) \quad \text { for } x \in \mathbb{R}^{n} \backslash B\left(0,2 R_{0} / \varepsilon\right) .
$$

By $(17)$ and $(18)$, we see that $\int_{\mathbb{R}^{n}} \chi_{\varepsilon}\left(u_{\varepsilon}\right)^{p+1} d x<1$ for sufficiently small $\varepsilon>0$.

The first property of (2) is proven in Lemma 8 . The second property of (2) can be proved in the same way with the arguments of [9]. The decaying property (3) follows from $(17)$ and $(18)$. From $(3)$, we see that the solution $u_{\varepsilon} \in E_{\varepsilon}$ belongs to $L^{2}\left(\mathbb{R}^{n}\right)$. This implies that $u_{\varepsilon} \in W^{1,2}\left(\mathbb{R}^{n}\right)$.

For the case $p \geq(n+2) /(n-2)$, we make the following modifications in the proofs. We define $f(u)=u^{p}$ for $|u| \leq 1$ and $f(u)=u^{q}$ for $|u| \geq 1$, where $1<q<$ $(n+2) /(n-2)$ is fixed. Then we consider (4) with $u^{p}$ replaced by $f(u)$. Setting $F(u)=$ $\int_{0}^{u} f(s) d s$, we consider

$$
M_{\varepsilon}=\inf \left\{\|u\|_{\varepsilon}^{2} \mid \int_{\mathbb{R}^{n}} F(u) d x=1, \int_{\mathbb{R}^{n}} \chi_{\varepsilon} F(u) d x \leq 1\right\} .
$$

Since $f$ is subcritical, $M_{\varepsilon}$ is achieved by some $u_{\varepsilon}$ which satisfies

$$
-\Delta u_{\varepsilon}+V(\varepsilon x) u_{\varepsilon}=\alpha_{\varepsilon} f(u)+\beta_{\varepsilon} \chi_{\varepsilon} f(u), \quad u_{\varepsilon}>0 .
$$

Lemma 5 still holds since we may use functions of small $L^{\infty}$ norms. Lemma 7 can be proved by modifying the proofs and by noticing that $F(u) \leq \frac{1}{q+1}|u|^{q+1}$. Lemma 8 is proved by the same arguments since $f(u) \leq u^{q}$. Then following the proofs for the subcritical case we deduce that $u_{\varepsilon}$ is a solution with $\beta_{\varepsilon}=0$. Then we can show $\left\|u_{\varepsilon}\right\|_{L^{\infty}} \rightarrow 0$ as $\varepsilon \rightarrow 0$, therefore $u_{\varepsilon}$ is a solution of the original equation. The rest of the proof is similar to that for the subcritical case.

This completes the proof of Theorem 1

We finish with some remarks for further results with details omitted. 
Remark 9. Our methods may be modified easily to construct solutions concentrating on lower dimensional spheres of zeroes of the potential when the potential $V(x)$ is radially symmetric with respect to some spaces. For example, $V(x)=V\left(x_{1}, x_{2}\right)$ with $\left(x_{1}, x_{2}\right) \in$ $\mathbb{R}^{n}=\mathbb{R}^{k_{1}} \times \mathbb{R}^{k_{2}}$, and $V\left(x_{1}, x_{2}\right)=V\left(\left|x_{1}\right|,\left|x_{2}\right|\right)$. We mention [7, 11, 12, 15, 16] for some related problems on solutions with multidimensional concentrations.

Remark 10. Our results cover potentials $V$ which stay away from zero at infinity: $\liminf _{|x| \rightarrow \infty} V(x)>0$. In this case, the solutions constructed are of exponential decay at infinity. In fact, as long as $\liminf _{|x| \rightarrow \infty}|x|^{\alpha} V(x)>0$ is satisfied for some $\alpha<2$, the solutions have exponential decay at infinity.

Remark 11. Spike solutions concentrating near zeroes of the potentials are obtained in [6] for a related problem with decaying potentials: $-\varepsilon^{2} \Delta v+V(x) v=K(x) v^{p}$ in $\mathbb{R}^{n}$ where the decaying rates for $V$ and $K$ are related and restricted by $p$. Using the methods in the present paper, condition $(V)$ may be sufficient for constructing spike solutions concentrating near zeroes of $V$.

Acknowledgements. The authors are grateful to Professor A. Ambrosetti for some discussions on related issues inspiring the current work. The authors thank the referee for some helpful suggestions which improved the presentation of the paper. The work of the first author was supported by grant No. R01-2004-000-10055-0 from the Basic Research Program of the Korea Science \& Engineering Foundation.

\section{References}

[1] Ambrosetti, A., Felli, V., Malchiodi, A.: Ground states of nonlinear Schrödinger equations with potentials vanishing at infinity. J. Eur. Math. Soc. 7, 117-144 (2005) Zbl 1064.35175 MR 2120993

[2] Ambrosetti, A., Malchiodi, A.: Perturbation Methods and Semilinear Elliptic Problems on $\mathbb{R}^{n}$. Progr. Math. 240, Birkhäuser (2006) Zbl pre05013399 MR 2186962

[3] Ambrosetti, A., Malchiodi, A., Ni, W.-M.: Singularly perturbed elliptic equations with symmetry: existence of solutions concentrating on spheres. I. Comm. Math. Phys. 235, 427-466 (2003) Zbl 1072.35019 MR 1974510

[4] Ambrosetti, A., Malchiodi, A., Ruiz, D.: Bound states of nonlinear Schrödinger equations with potentials vanishing at infinity. J. Anal. Math., to appear

[5] Ambrosetti, A., Ruiz, D.: Radial solutions concentrating on spheres of NLS with vanishing potentials. Preprint

[6] Ambrosetti, A., Wang, Z.-Q.: Nonlinear Schrödinger equations with vanishing and decaying potentials. Differential Integral Equations 18, 1321-1332 (2005) MR 2174974

[7] Bartsch, T., Peng, S.: Semiclassical symmetric Schrödinger equations: existence of solutions concentrating simultaneously on several spheres. Preprint

[8] Byeon, J.: Existence of large positive solutions of some nonlinear elliptic equations on singularly perturbed domains. Comm. Partial Differential Equations 22, 1731-1769 (1997) Zbl 0883.35040 MR 1469588

[9] Byeon, J., Wang, Z.-Q.: Standing waves with a critical frequency for nonlinear Schrödinger equations. Arch. Rat. Mech. Anal. 165, 295-316 (2002) Zbl 1022.35064 MR 1939214 
[10] Byeon, J., Wang, Z.-Q.: Standing waves with a critical frequency for nonlinear Schrödinger equations, II. Calc. Var. Partial Differential Equations 18, 207-219 (2003) Zbl 1073.35199 MR 2010966

[11] Cerami, G., Passaseo, D.: The effect of concentrating potentials in some singularly perturbed problems. Calc. Var. Partial Differential Equations 17, 257-281 (2003) Zbl pre01969061 MR 1989833

[12] Dancer, E. N., Yan, S.: A new type of concentration solutions for a singularly perturbed elliptic problem. Preprint

[13] Floer, A., Weinstein, A.: Nonspreading wave packets for the cubic Schrödinger equation with a bounded potential. J. Funct. Anal. 69, 397-408 (1986) Zbl 0613.35076 MR 0867665

[14] Gilbarg, D., Trudinger, N. S.: Elliptic Partial Differential Equations of Second Order. 2nd ed., Grundlehren Math. Wiss. 224, Springer, Berlin (1983) Zbl 0562.35001 MR 0737190

[15] Malchiodi, A., Montenegro, M.: Boundary concentration phenomena for a singularly perturbed elliptic problem. Comm. Pure Appl. Math. 55, 1507-1568 (2002) Zbl pre01860559 MR 1923818

[16] Molle, R., Passaseo, D.: Concentration phenomena for solutions of superlinear elliptic problems. Ann. Inst. H. Poincaré Anal. Non Linéaire, to appear 